\title{
Analyse multivariable de quelques caractères morphologiques de populations de chênes (Quercus robur L. et Quercus petraea (Matt.) Liebl.) du Hurepoix
}

\author{
J.L. DUPOUEY (*) \\ Laboratoire de Phyto-écologie forestière \\ I.N.R.A., Centre de Recherches forestières, Champenoux, F 54280 Seichamps
}

\begin{abstract}
Résumé
L'auteur analyse la variabilité des caractères morphologiques des feuilles, infrutescences et écorces dans deux populations de chênes sessiles et pédonculés sympatriques. Quinze caractères, divisés en un total de cinquante-trois classes sont ainsi mesurés pour quarante-trois arbres. L'analyse factorielle des correspondances, qui apparaît comme la méthode la plus objective, permet de distinguer nettement les individus à rapporter au taxon Quercus petraea de ceux appartenant à Quercus robur. Les caractères discriminants sont la longueur du pédoncule, la longueur du pétiole et l'angle des oreillettes. Glands, cupules et écorces ne sont pas significativement différents, pour les caractères étudiés, dans les deux espèces. La présence de moins de $5 \%$ d'arbres morphologiquement intermédiaires dénote de la faiblesse des phénomènes d'hybridation-introgression dans cet échantillon. La généralité de ce résultat est discutée.
\end{abstract}

\section{Introduction}

Si par le passé, le chêne sessile (Quercus petraea (Matt.) Liebl.) et le chêne pédonculé (Quercus robur L.) ont souvent été réunis dans la gestion forestière, il apparaît aujourd'hui nécessaire de leur appliquer des traitements sylvicoles différents, en accord avec leurs exigences différentes. Dès 1897, MATHIEU souligne dans sa flore forestière : «On ne saurait attacher trop d’importance, dans les travaux de repeuplement, à cette différence fondamentale, afin de placer chacune de ces deux essences dans la station qui lui est propre ». Le dépérissement du chêne en forêt de Tronçais (Allier), qui scmble n'affecter que le pédonculé à l'exclusion du sessile (BECKER \& LevY, 1982), montre lacuité d'une telle réflexion et la nécessité qu'il y a encore aujourd'hui d'approfondir nos connaissances sur l'écologie respective des deux espèces.

Or les études autécologiques ne peuvent être dissociées de celles du statut taxonomique du matériel végétal étudié. On sait par exemple les difficultés souvent rencontrées

(*) Cette étude a été réalisée avec les étudiants et enseignants du D.E.A. d'Ecologie de la Faculté d'Orsay de l'année 1980-1981. 
par les phytosociologues lors des relevés de végétation où les deux espèces de chênes sont présentes. Dans le cas du chêne, ces études taxonomiques ont divisé les botanistes sur une question non encore résolue : les individus morphologiquement intermédiaires entre chêne sessile et chêne pédonculé, très fréquemment observés dans la nature, sont-ils le fait de la grande variabilité de l'une ou l'autre des deux espèces, ou le résultat de phénomènes d'hybridation et d'introgression? On trouvera une analyse de cette controverse dans un article de Gardiner (1970).

Dans le cadre de l'étude autécologique des deux chênes, menée dans quelques stations du Hurepoix (rebord oriental du plateau de Beauce), nous avons, dans un premier temps, étudié la variabilité de quelques caractères morphologiques en essayant de préciser:

- le statut taxonomique des individus étudiés (chêne sessile, chêne pédonculé, ou hybride), observée,

- les caractères morphologiques discriminants responsables de la variabilité ceci sur la base d'une analyse multivariable des données.

\section{Site, matériel et méthode}

Les chênes ont été échantillonnés au cours du mois de septembre dans deux zones forestières du Hurepoix (région soumise à une variante sèche du macroclimat du Bassin Parisien, où le hêtre est sporadique).

\subsection{Sur le campus de la Faculté des Sciences d'Orsay}

Le long d'un transect large de $200 \mathrm{~m}$, établi sur la rive gauche de l'Yvette (exposée au Sud), la presque totalité des chênes a été inventoriée - soit environ cent cinquante individus - Après détermination sur le terrain d'après les critères usuels, nous avons obtenu les proportions suivantes : 27 p. 100 de chênes sessiles, 33 p. 100 de chênes pédonculés et 40 p. 100 d'individus que nous avons qualifiés provisoirement d' "hybrides » en raison de leurs caractères morphologiques intermédiaires. Trente-quatre chênes ont ensuite été choisis pour le prélèvement des échantillons de manière à :

- couvrir de façon la plus homogène possible lensemble du transect ;

- avoir un nombre sensiblement égal d'individus de chacune des trois catégories précédentes ;

- éviter les chênes trop rapprochés; en effet, les échantillons ayant été ramassés au sol, il fallait être sûr de leur provenance.

Ces conditions définissent en fait un échantillonnage stratifié selon le milieu et l'appartenance taxonomique définie a priori.

Les caractéristiques topographiques et géologiques du transect sont présentées figure 1. Les sols du versant sont pour la plupart développés sur un matériau colluvionnaire d'origine périglaciaire et dont la composition présente des proportions variées de chacune des formations en place. Ce sont des sols bruns, bruns lessivés ou podzoliques, présentant divers degrés d'hydromorphie selon la pente. 


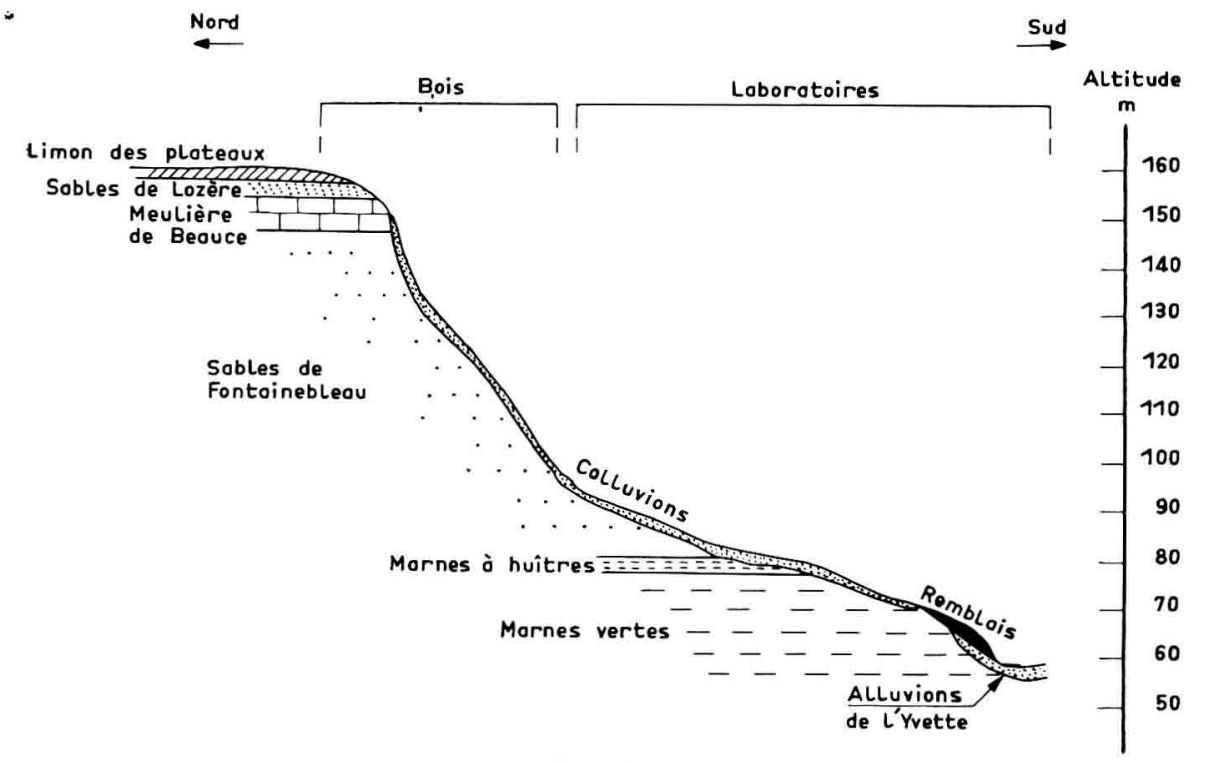

FIG. 1

Coupe géologique schématique du transect étudié.

Geological sectional scheme of the studied transect.

L'étude phytosociologique (BAUI.E, 1980) montre la présence de groupements du Carpinion betulii Oberdorfer 1953 et du Quercion rohori-petraeae (Malcuit, 1929) Br.-B1. 1932.

\subsection{Sur le plateau de Hurepoix (autour de Boullay-les-Troux)}

Neuf chênes ont été choisis, dans des stations semblables aux précédentes.

Pour chaque chêne inventorié, on a ramassé au sol feuilles et fruits jusqu'à concurrence d'au moins vingt-cinq échantillons (bien que RusHTon, 1978, scmble avoir montré que cinq échantillons par arbre suffisent, du moins pour les feuilles). Toutefois, pour certains chênes pédonculés, ce nombre de fruits n'a pu être atteint, la glandée ayant été inférieure en abondance à celle du chêne sessile. Becker (1972), Pellecuer (1976), TurBang (1954), font une observation analogue. JONEs (1959) constate pour sa part, après plusieurs années d’études, une fertilité égale des deux espèces: Poskin (1934), quant à lui, observe une production supérieure du chêne pédonculé. I! semble donc impossible actuellement de généraliser de telles observations. La glandée de 1980 a toutefois été suffisante pour que nous ne soyons pas obligés d'écarter de l'échantillon les arbres à faible production.

De plus, nous avons mesuré sur l'arbre certaines variables concernant le rhytidome, présentées ci-dessous.

Notons enfin que l'origine «naturelle» sinon spontanée de ces peuplements, bien que vraisemblable, ne peut être assurée. 


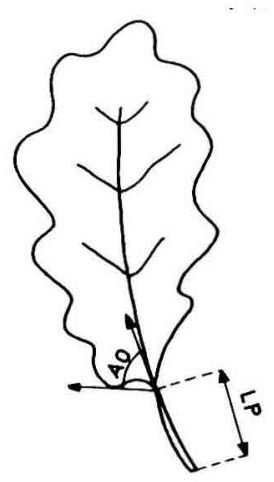

a. La feuille

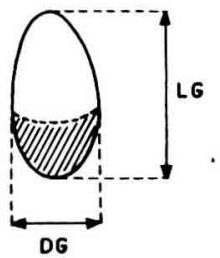

d. Le gland

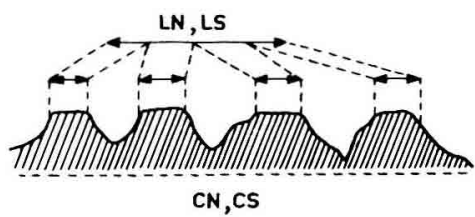

b. L'écorce

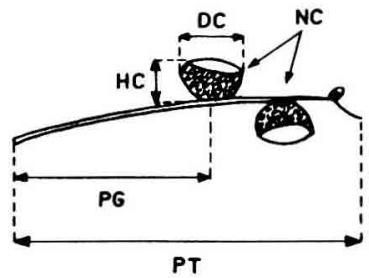

c. L'infrutescence

FIG. 2

Les caractères morphologiques mesurés.

The morphological characters assessed.

\section{Caractères mesurés}

De très nombreux caractères ont été proposés pour différencier les deux espèces. PosKIN (1934) en fait une présentation relativement exhaustive.

Nous avons choisi pour notre part des caractères facilement mesurables (éliminant par exemple les caractères floraux, ainsi que ceux concernant le bois ou le port), limitant au maximum la subjectivité de l'observation (mesures quantitatives) et susceptibles de discriminer les deux espèces. De nombreux caractères, peut-être plus discriminants, auraient pu être inclus dans létude (nombre de lobes foliaires, position de la plus grande largeur des feuilles...). Mais la multiplication des mesures aurait rendu une telle étude trop lourde, sans garantir de meilleurs résultats.

Ont été retenus pour l'analyse (voir fig. 2) :

\subsection{Pour la feuille}

- longueur du pétiole (LP) ;

- angle des oreillettes (AO). 
Sur chaque feuille, on mesure l'angle de la tangente au lobe le plus développé passant par le point de jonction du limbe au pétiole avec l'axe de la feuille.

Cette méthode a été adoptée afin d'éliminer les notations subjectives comme par exemple la comparaison avec des séries de feuilles typiques, utilisée par Rushton (1974) ou KISSLING (1980 a).

\subsection{Pour les fruits}

- longueur totale du pédoncule, du point d'attachement au rameau jusqu'à l'extrémité de l'infrutescence (PT) ;

- longueur du pédoncule jusqu'à la première cupule (PG), ceci afin de supprimer le biais éventuel introduit lors de la mesure de PT par les pédoncules brisés;

- nombre de cupules (développées) par pédoncule (NC) ;

- hauteur des cupules (HC);

- diamètre des cupules (DC) ;

- Longueur des glands (LG) ;

- diamètre maximum des glands (DG).

\subsection{Pour l'écorce}

Nous avons appliqué sur l'écorce de l'arbre, à la hauteur de $1,30 \mathrm{~m}$, deux arcs de cercle d'une longueur de $30 \mathrm{~cm}$ chacun, l'un sur la face exposée au Nord, l'autre sur la face exposée au Sud.

Ont été mesurés le long de ces ares :

- le nombre de côtes du rhytidome (CN au Nord et CS au Sud);

- la largeur cumulée du sommet des côtes (LN et LS).

Au total donc, quatre caractères de l'écorce, sept de linfrutescence et deux des feuilles sont pris en compte. Le but étant surtout de déterminer des critères simples de différenciation, s'ils existent, nous avons éliminé de l'analyse toutes les variables synthétiques obtenues par combinaison des précédentes (excentricité du gland...). Comme le souligne Rushton (1978), il n'y a pas de raison a priori de supposer que les caractères bruts donnent une mauvaise séparation taxonomique.

\section{L'analyse des données}

Les différentes méthodes utilisées dans l'analyse des données morphologiques pour les espèces morphologiquement proches sont décrites par WigSToN (1974). Parmi celles-ci, deux ont été particulièrement employées pour l'étude des populations de chênes. 


\subsection{La méthode des diagrammes de dispersion symboliques} (ou métroglyphes)

Sur un plan repéré par deux axes, on reporte la position des individus étudiés selon leurs valeurs pour deux variables choisies (par exemple, longueur du pédoncule floral sur l'axe des abscisses et longueur du pétiole sur l'axe des ordonnées - voir fig. 3). Sur ce diagramme de dispersion à deux variables, d'autres caractères sont figurés par des symboles ajoutés sur chaque point représentant un individu. On obtient alors un métroglyphe. La position respective des points permet de décider s'il s'agit de populations pures, hybrides, introgressées...

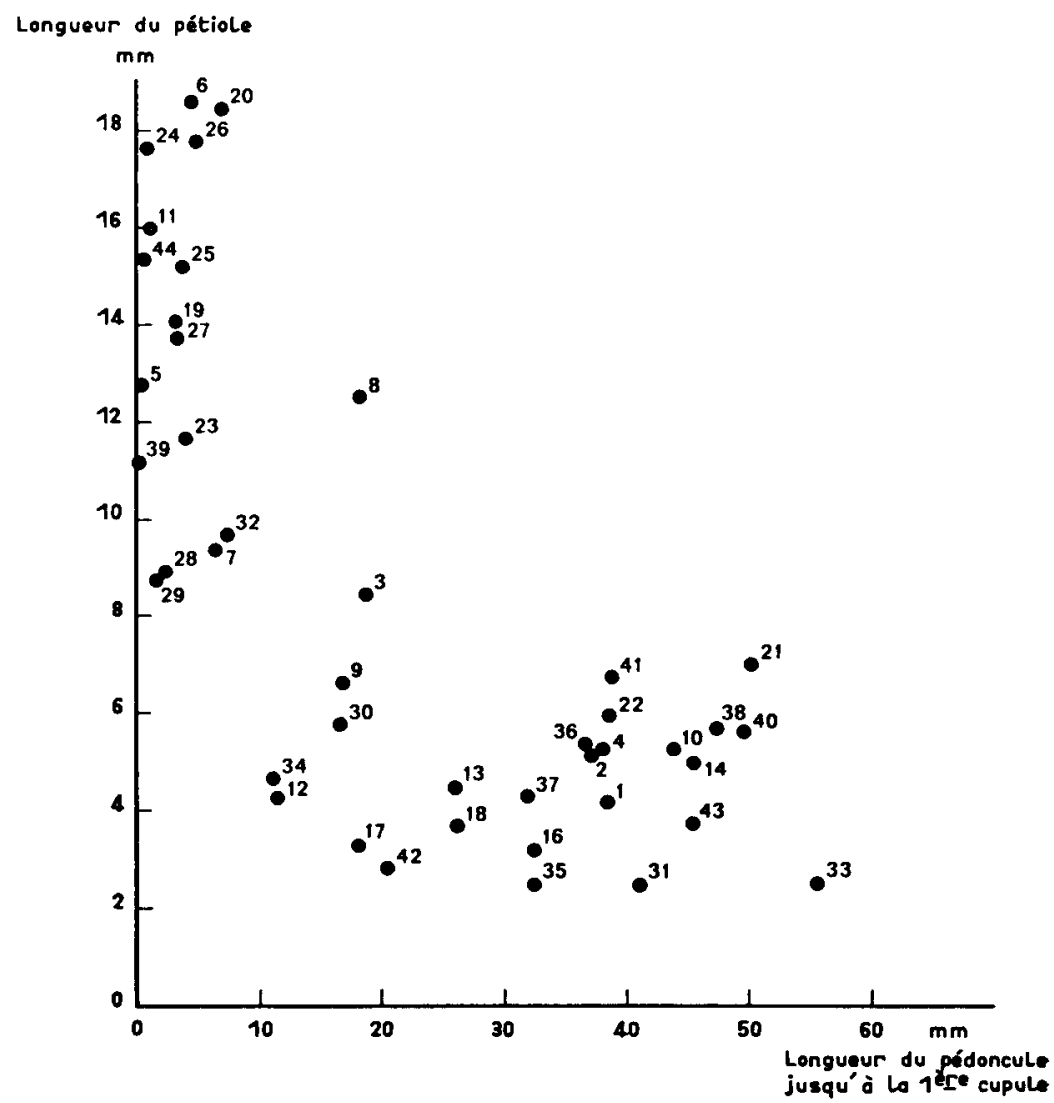

FIG. 3

Diagramme de dispersion à deux variables

- abscisse : longueur moyenne du pédoncule par arbre,

- ordonnée : longueur moyenne du pétiole par arbre.

Bivariate scatter diagram showing the relationship between mean peduncle length per tree (absciss) and mean petiole length per tree (ordinate). 
Rushton (1978) a très bien montré les dangers du choix a priori de deux variables descriptives, auxquelles on accorde de façon subjective une importance particulière. Ce même auteur a, par contre, recours à l'autre méthode, pourtant non exempte de subjectivité comme nous allons le montrer.

\subsection{L'indice d'hybridité}

Chaque caractère étudié est noté 0 s'il se rapporte, dans le cas des chênes, à Quercus robur, 1 s'il est «typique» de Quercus petraea et $\frac{1}{2}$ s'il est de type intermédiaire (ou encore 0 pour Quercus robur, 4 pour Quercus petraea et de 1 à 3 pour les hybrides). Pour chaque individu, on fait la somme des notes pour tous les caractères étudiés. Ainsi, si on étudie n caractères, un pédonculé type aura un indice 0 , un rouvre typique un indice $\mathrm{n}$ et les «hybrides» des indices d'hybridité entre 0 et $n$, selon le degré d'introgression.

$$
\mathrm{IH}=\mathrm{n}_{\mathrm{r}} \times 0+\mathrm{n}_{\mathrm{j}} \times \frac{1}{2}+\mathrm{n}_{\mathrm{p}} \times 1
$$

IH : indice d'hybridité

$\mathrm{n}_{\mathrm{r}}$ : nombre de caractères attribués à Quercus robur

$\mathrm{n}_{\mathrm{p}}$ : nombre de caractères attribués à Quercus petraea

$\mathrm{n}_{\mathbf{i}}$ : nombre de caractères intermédiaires

Supposons maintenant que certains caractères considérés comme intermédiaires soient en fait à attribuer à l'une ou l'autre des espèces :

d'où

$$
\mathrm{IH}^{\prime}=\left(\mathrm{n}_{\mathrm{r}}+\mathrm{e}\right) \times 0+\left(\mathrm{n}_{\mathrm{i}}-\mathrm{e}-\mathrm{e}^{\prime}\right) \times \frac{1}{2}+\left(\mathrm{n}_{\mathrm{p}}+\mathrm{e}^{\prime}\right) \times 1
$$

$$
\mathbf{I} \mathbf{H}^{\prime}=\mathbf{I H}+\frac{1}{2}\left(\mathrm{e}^{\prime}-\mathrm{e}\right)
$$

e : nombre de caractères attribués à tort au type intermédiaire et faisant partie du domaine de Quercus robur

$\mathrm{e}^{\prime}$ : nombre de caractères attribués à tort au type intermédiaire et faisant partie du domaine de Quercus petraea

On constate donc que l'index d'hybridité augmente la proportion d'index de type «hybride» selon les critères de choix de la nature intermédiaire ou pure d'un caractère.

La critique principale de cette méthode est donc qu'on y choisit les caractères hybrides avant l'étude.

\subsection{L'analyse factorielle des correspondances}

Nous avons choisi une analyse multivariable qui assure la meilleure « objectivité * au départ.

Les caractères mesurés ont été divisés en un certain nombre de classes (tabl. 1), délimitées comme suit : pour chaque caractère, on choisit d'abord le nombre de classes en fonction de la variabilité totale. Les limites des classes sont alors déterminées de façon à ce que chacune contienne le même effectif d'échantillons (ce qui minimise la perte d'information). L'analyse factorielle des correspondances se rapporte donc à la matrice 
contenant pour chaque arbre (individus) et pour chaque classe de caractère (variables) la valeur 1 si l'arbre appartient à cette classe, la valeur 0 sinon.

\section{Tableau 1}

Variables de l'analyse factorielle.

Characters and classes used in the reciprocal averaging.

\begin{tabular}{|c|c|c|c|}
\hline \multicolumn{2}{|c|}{ Longueur du pédoncule (PT et PG) } & \multicolumn{2}{|c|}{ Diamètre de la cupule (DC) } \\
\hline & & \multicolumn{2}{|c|}{ DC $1 \quad 6-13(\mathrm{~mm})$} \\
\hline PT 1 & $0-5(\mathrm{~mm})$ & \multicolumn{2}{|c|}{ DC 2. $\quad 13-15$} \\
\hline PT 2 & $5-10$ & \multicolumn{2}{|c|}{ DC $3 \quad 15-17$} \\
\hline PT 3 & $10-20$ & \multirow{3}{*}{\multicolumn{2}{|c|}{ DC $4 \quad 17-22$}} \\
\hline PT 4 & $20-40$ & & \\
\hline PT 5 & $40-60$ & & \\
\hline PT 6 & $60-130$ & \multicolumn{2}{|c|}{ Longueur du gland (LG) } \\
\hline PG 1 & $0-2(\mathrm{~mm})$ & \multicolumn{2}{|c|}{ LG $1 \quad 8-16(\mathrm{~mm})$} \\
\hline PG 2 & $2-5$ & \multicolumn{2}{|c|}{ LG $2 \quad 16-20$} \\
\hline PG 3 & $5-15$ & \multirow{2}{*}{\multicolumn{2}{|c|}{$\begin{array}{ll}\text { LG } 3 & 20-24 \\
\text { LG } 4 & 24-38\end{array}$}} \\
\hline PG 4 & $15-30$ & \multirow{3}{*}{\multicolumn{2}{|c|}{ LG $4 \quad 24-38$}} \\
\hline PG 5 & $30-44$ & & \\
\hline PG 6 & 44 et plus & & \\
\hline \multicolumn{2}{|c|}{ Longueur pétiole (LP) } & \multicolumn{2}{|c|}{ Diamètre du gland (DG) } \\
\hline LP 1 & $1-5(\mathrm{~mm})$ & \multirow{4}{*}{$\begin{array}{l}\text { DG } 1 \\
\text { DG } 2 \\
\text { DG } 3\end{array}$} & $5-12(\mathrm{~mm})$ \\
\hline LP 2 & $5-9$ & & $\begin{array}{l}12-15 \\
15-20\end{array}$ \\
\hline LP 3 & $9-15$ & & $15-29$ \\
\hline LP 4 & $15-30$ & & \\
\hline \multicolumn{2}{|c|}{ Angle des oreillettes (AO) } & \multicolumn{2}{|c|}{ Nombre de côtes (CN et CS) } \\
\hline AO 1 & 30- 70 (degrés sexagésimaux) & $\mathrm{CN} 1$ & $0 \quad-0,27$ (nombre de côtes $/ \mathrm{cm}$ ) \\
\hline $\mathrm{AO} 2$ & $70-100$ & $\mathrm{CN} 2$ & $0,27-0,33$ \\
\hline $\mathrm{AO}_{3}$ & $100-140$ & \multirow{2}{*}{\multicolumn{2}{|c|}{$0,41-1$}} \\
\hline $\mathrm{AO} 4$ & $140-180$ & & \\
\hline \multirow{2}{*}{\multicolumn{2}{|c|}{ Nombre de cupules par pédoncule (NC) }} & $\mathrm{CS} 1$ & $0 \quad-0,27$ (nombre de côtes $/ \mathrm{cm}$ ) \\
\hline & & $\operatorname{CS} 2$ & $0,27-0,33$ \\
\hline $\mathrm{NC} 1$ & 1 & CS 3 & $0,33-0,39$ \\
\hline $\mathrm{NC} 2$ & 2 & $\operatorname{CS} 4$ & $0,39-1$ \\
\hline $\mathrm{NC} 3$ & 3 & & \\
\hline \multirow[t]{2}{*}{$\mathrm{NC} 4$} & 4 et plus & & \\
\hline & & \multicolumn{2}{|c|}{ Longueur des côtes (LN et LS) } \\
\hline Haute & de la cupule (HC) & LN 1 & $0 \quad-0,43(\mathrm{~cm} / \mathrm{cm})$ \\
\hline HC 1 & 3- $7(\mathrm{~mm})$ & LN 2 & $0,43-0,49$ \\
\hline $\mathrm{HC} 2$ & $7-8$ & LN 3 & $0,49-1$ \\
\hline $\mathrm{HC} 3$ & 8- 9 & & \\
\hline $\mathrm{HC} 4$ & $9-11$ & LS 1 & $0 \quad-0,43(\mathrm{~cm} / \mathrm{cm})$ \\
\hline HC 5 & $11-19$ & LS 2 & $0,43-0,51$ \\
\hline
\end{tabular}




\section{Tableau 2}

Pourcentage de l'inertie totale expliquée par chaque axe.

Pourcentage of the total variance accounted for by each component.

\begin{tabular}{c|c|c}
\hline \hline Axe & $\begin{array}{c}\% \\
\text { de la variance totale }\end{array}$ & Histogramme des valeurs propres \\
\hline 1 & 23,9 & \\
2 & 10,6 & \\
3 & 9,8 & \\
4 & 9,2 & \\
5 & 6,7 & \\
\hline
\end{tabular}

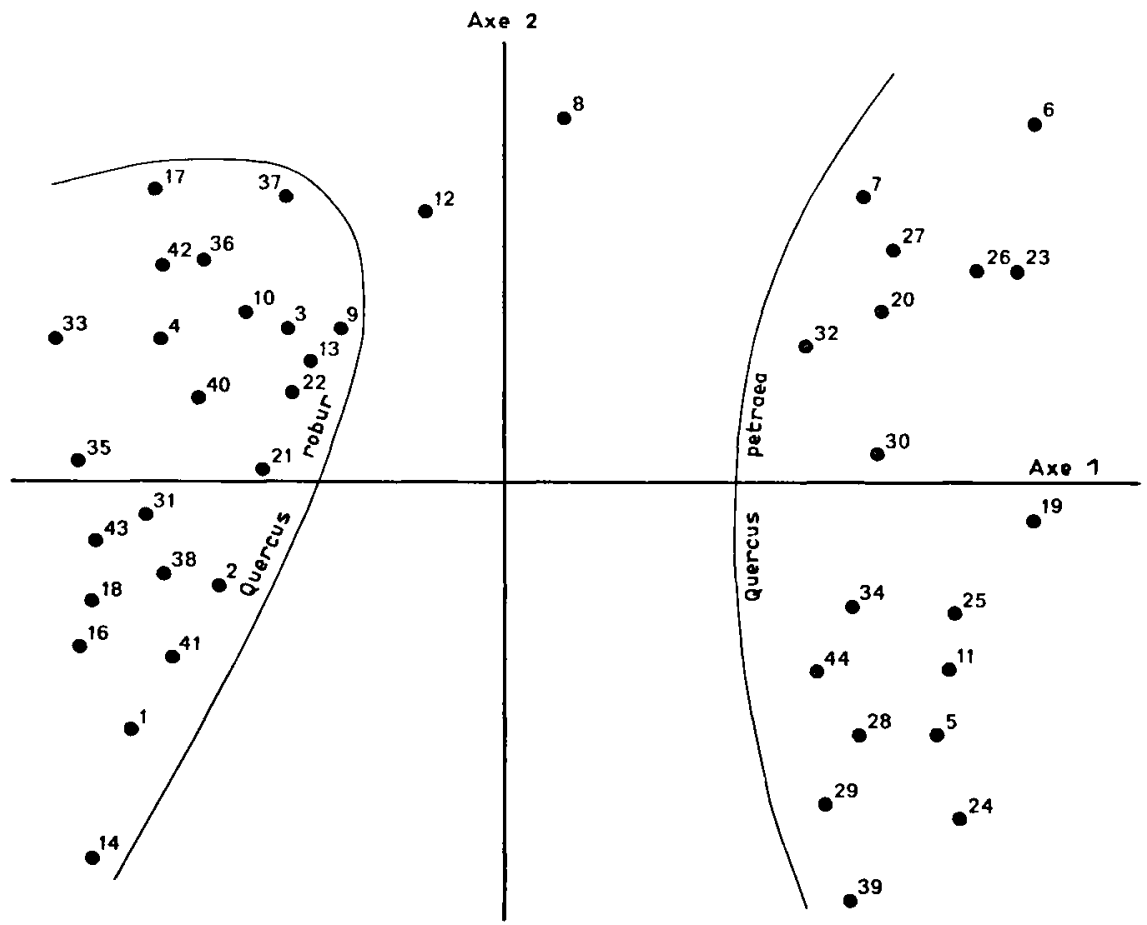

FIG. 4

Individus dans le plan des axes 1-2 de l'analyse factorielle des correspondances (A.F.C.). Les lignes regroupent les individus «typiques 》 de chaque espèce.

Trees in the plan of the first two components of the reciprocal averaging (R.A.). Lines gather trees typical of each species. 


\section{Résultats}

Le pourcentage de la variance totale expliqué par chaque axe est donné au tableau 2. On constate que le premier axe recèle 23,8 p. 100 de la variance totale, les suivants décroissant régulièrement à partir de 10,5 p. 100. Il y a donc séparation nette du premier axe.

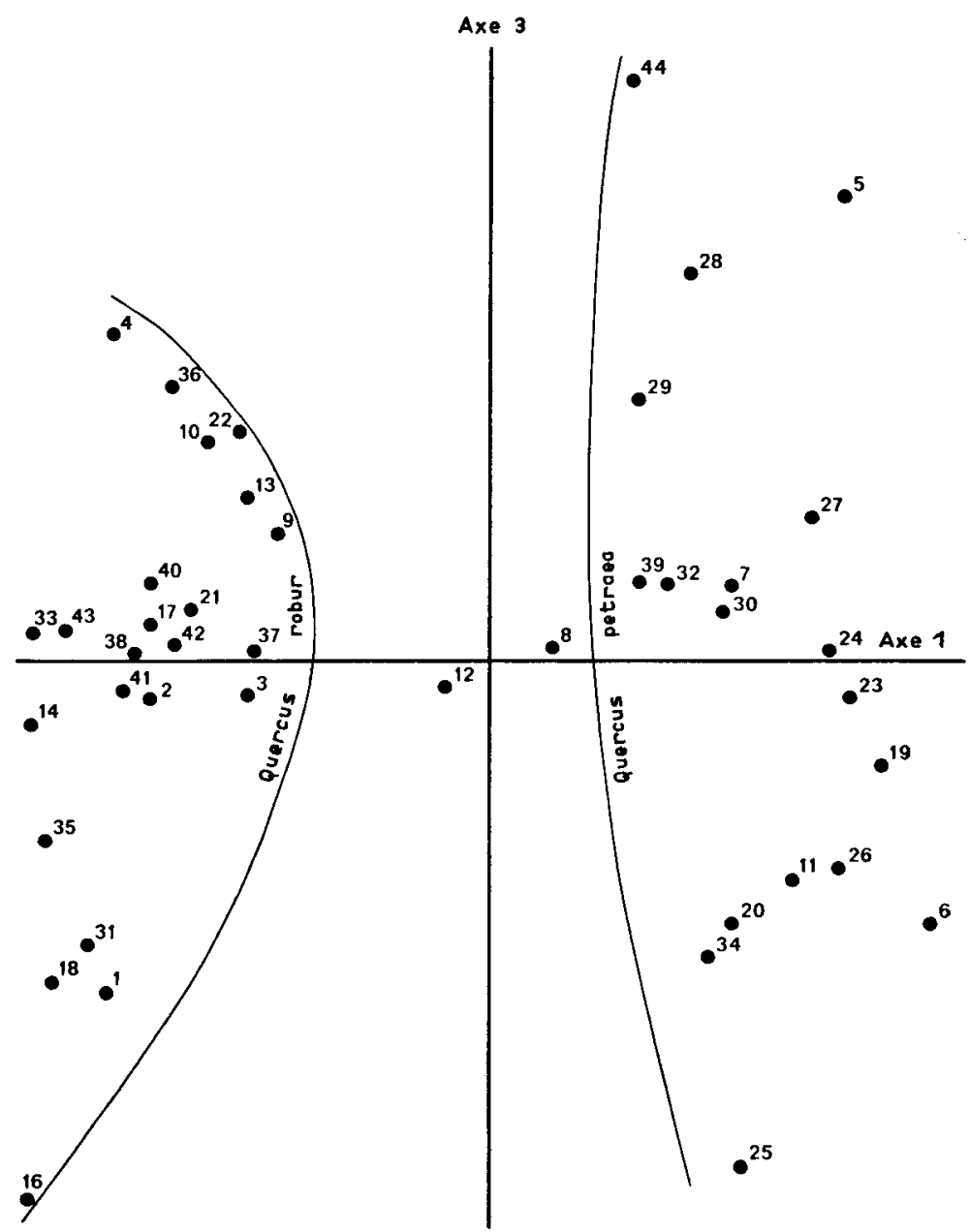

FIG. 5

Individus dans le plan des axes $1-3$ de l'A.F.C.

Trees in the plan of the first and third components of R.A. 


\subsection{Les individus}

On trouvera figures 4 et 5 les résultats de l'analyse pour les axes 1-2 et 1-3. Il y a une différenciation nette de deux ensembles le long de l'axe 1, les abscisses positives correspondant aux chênes sessiles, les négatives aux chênes pédonculés. On trouve deux individus en position intermédiaire, les arbres $n^{0 *} 12$ et 8 . Par contre, le long des axes 2 et 3 , il n'est pas possible d'établir des discontinuités à l'intérieur des deux sous-ensembles précédents, et ceci se retrouve le long des axes suivants (étudiés jusqu'au cinquième).

\subsection{Les variables}

Les résultats dans le plan des axes factoriels 1-2 et 1-3 sont figurés figures 6 et 7 .

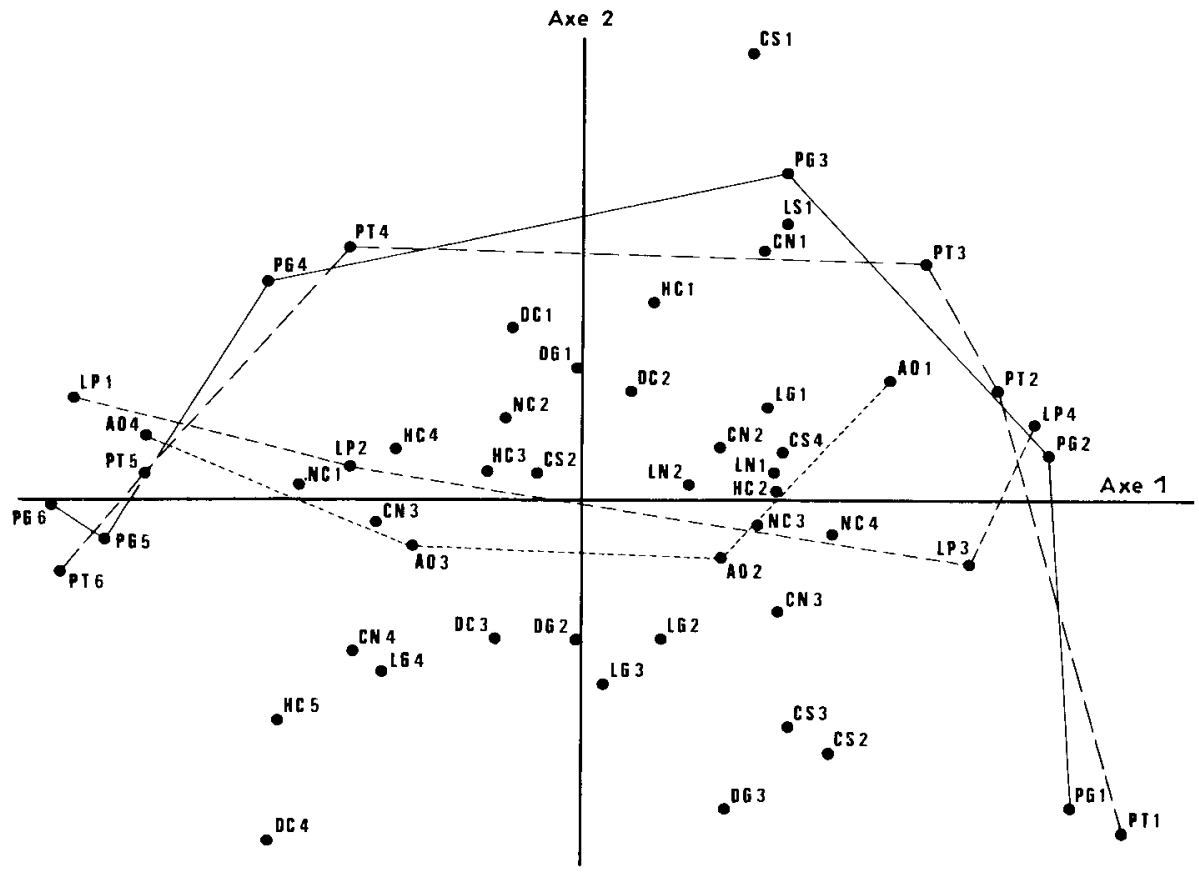

FIG. 6

Variables dans le plan des axes 1-2 de l'A.F.C.

Les lignes joignent les classes des variables les plus significatives.

Characters in the plan of the first two components of the R.A.

Lines join the classes of significant characters together.

L'étude de la contribution absolue de chaque variable pour les trois premiers axes (en pourcentage de linertie totale du nuage) permet de hiérarchiser les caractères responsables de la formation de chaque axe (tabl. 3). Sur les axes 2 et 3 , on ne distingue aucun gradient morphologique capable d'expliquer la répartition des arbres. Sur le premier axe, qui semble donc être le seul interprétable, les caractères PG et PT (longueur du pédoncule), LP (longueur du pétiole) et AO (angle des oreillettes) se détachent nettement. 


\section{TABLEAU 3}

Contribution absolue des variables aux trois premiers axes.

Absolute contribution of characters to the first three components.

\begin{tabular}{|c|c|c|c|}
\hline Variable & Axe 1 & Axe 2 & Axe 3 \\
\hline PG $1 \ldots \ldots \ldots \ldots$ & $59 \%$ & $101 \%$ & $68 \%$ \\
\hline PG $2 \ldots \ldots \ldots$ & 76 & $a$ & 28 \\
\hline PG $3 \quad \ldots \ldots \ldots$ & 17 & 98 & 21 \\
\hline PG $4 \ldots \ldots \ldots \ldots$ & 24 & 27 & 6 \\
\hline PG $5 \quad \ldots \ldots \ldots \ldots$ & 53 & 1 & 0 \\
\hline PG $6 \quad \ldots \ldots \ldots \ldots$ & 53 & 0 & 0 \\
\hline PG $\ldots \ldots \ldots \ldots$ & $28,2 \%$ & $22,7 \%$ & $12,3 \%$ \\
\hline PT $1 \ldots \ldots \ldots \ldots$ & $55 \%$ & $111 \%$ & $88 \%$ \\
\hline PT $2 \ldots \ldots \ldots \ldots$ & 53 & 5 & 17 \\
\hline PT $3 \ldots \ldots \ldots \ldots$ & 39 & 48 & 51 \\
\hline PT $4 \ldots \ldots \ldots \ldots$ & 12 & 41 & 2 \\
\hline PT $5 \ldots \ldots \ldots \ldots$ & 41 & 0 & 0 \\
\hline PT $6 \ldots \ldots \ldots \ldots$ & 67 & 3 & 0 \\
\hline PT $\ldots \ldots \ldots \ldots$ & $26,7 \%$ & $20,8 \%$ & $\overline{15,8 \%}$ \\
\hline LP $1 \ldots \ldots \ldots \ldots$ & $89 \%$ & $5 \%$ & $12 \%$ \\
\hline LP $2, \ldots \ldots \ldots \ldots$ & 7 & 0 & 19 \\
\hline LP $3 \ldots \ldots \ldots \ldots$ & 56 & 12 & 3 \\
\hline LP $4 \ldots \ldots \ldots \ldots$ & 68 & 3 & 18 \\
\hline$L P \ldots \ldots$ & $22 \%$ & $2,0 \%$ & $5,2 \%$ \\
\hline AO $1 \ldots \ldots \ldots$ & $52 \%$ & $12 \%$ & $26 \%$ \\
\hline $\mathrm{AO}_{2} \ldots \ldots \ldots \ldots$ & 6 & 2 & 9 \\
\hline $\mathrm{AO}_{3} \ldots \ldots \ldots$ & 2 & 3 & 30 \\
\hline $\mathrm{AO} 4 \ldots \ldots \ldots \ldots$ & 61 & 1 & 13 \\
\hline AO $\ldots \ldots \ldots \ldots$ & $12,1 \%$ & $1,8 \%$ & $7,8 \%$ \\
\hline HC $1 \ldots$ & $2 \%$ & $47 \%$ & $1 \%$ \\
\hline $\mathrm{HC} 2 \ldots \ldots \ldots \ldots$ & 9 & 1 & 8 \\
\hline $\mathrm{HC}_{3} \ldots \ldots \ldots \ldots$ & 1 & 0 & 0 \\
\hline $\mathrm{HC} 4 \ldots \ldots \ldots \ldots$ & 7 & 1 & 11 \\
\hline HC $5 \quad \ldots \ldots \ldots$. & 18 & 52 & 18 \\
\hline $\mathrm{HC} \ldots \ldots \ldots \ldots$ & $3,7 \%$ & $10 \%$ & $3,8 \%$ \\
\hline NC $1 \ldots \ldots \ldots$. & $10 \%$ & $0 \%$ & $10 \%$ \\
\hline $\mathrm{NC} 2 \ldots \ldots . . .$. & 1 & 7 & 1 \\
\hline $\mathrm{NC} 3 \ldots \ldots \ldots$ & 10 & 0 & 4 \\
\hline NC $4 \ldots \ldots \ldots$. & 12 & 1 & 11 \\
\hline $\mathrm{NC} \ldots \ldots$ & $3,3 \%$ & $0,8 \%$ & $2,6 \%$ \\
\hline
\end{tabular}


Tableau 3 (suite)

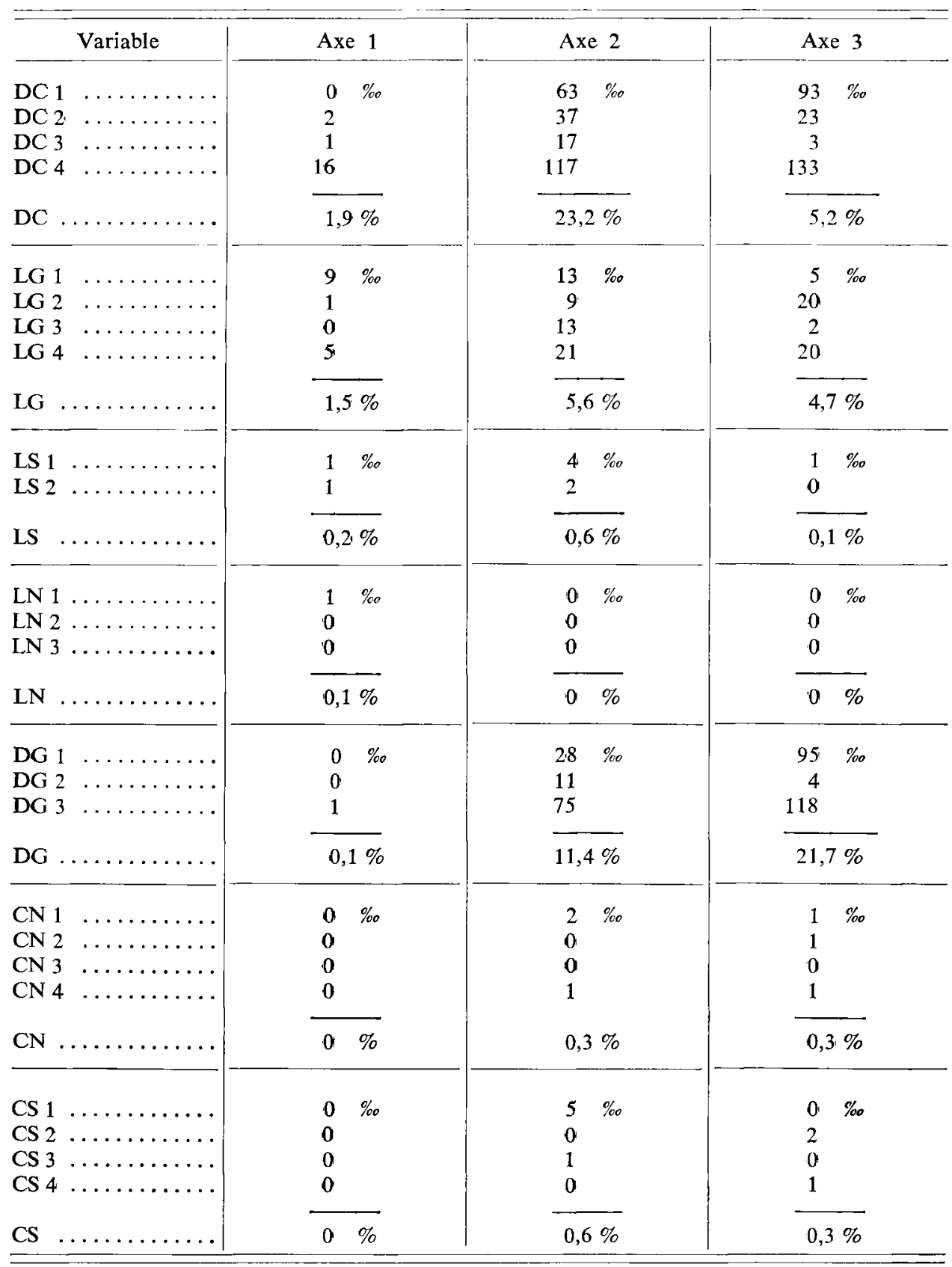




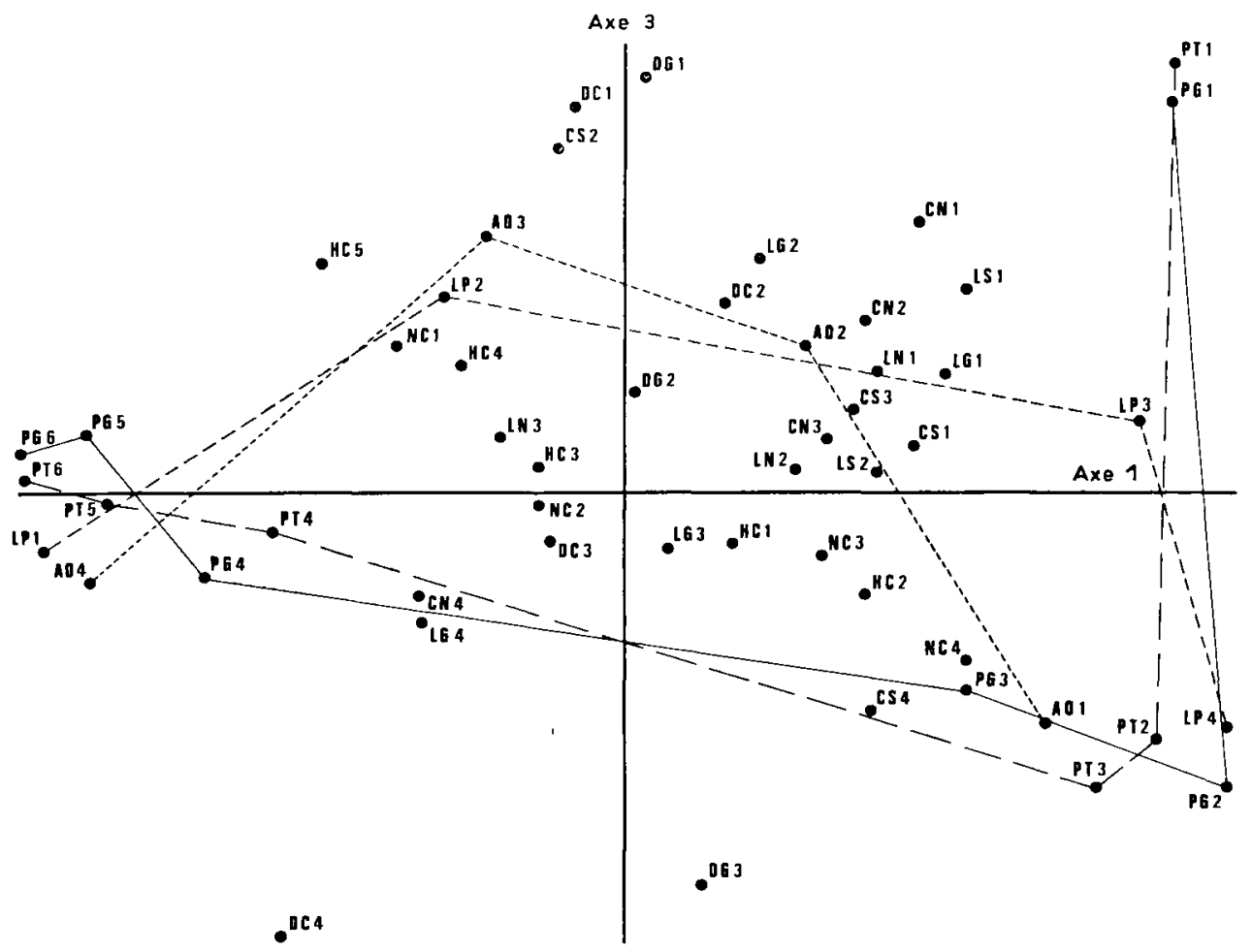

FIG. 7

Variables dans le plan des axes 1-3 de l'A.F.C.

Characters in the plan of the first and third components of R.A.

\section{Discussion}

Il est remarquable de constater, au sein des populations étudiées ici, une très bonne discrimination entre chêne sessile et chêne pédonculé, d'autant plus que lors du prélèvement des échantillons sur le terrain, il était souvent apparu difficile de rattacher les individus observés à l'une ou l'autre des deux espèces. La forme sensiblement parabolique du nuage de points dans le plan des axes 1 et 2 de l'analyse factorielle des correspondances est due à un effet Guttman, confirmant l'existence d'un facteur hautement significatif de la structure des données. En l'occurrence, ce facteur significatif correspond à la structure du génome des individus étudiés, plus ou moins proche de l'une ou l'autre espèce.

La séparation entre les deux espèces est si nette que le diagramme de la figure 3 peut être rapproché de celui représenté par Rushton (1978, fig. 8) dans lequel sont analysées deux populations, "typiques " de chaque espèce, mais allopatriques (alors que, rappelons-le, les individus que nous avons analysés sont, pour au moins trente-quatre d'entre eux, en mélange pied à pied). Seul deux individus peuvent encore être qualifiés 
d'intermédiaires pour l'ensemble des caractères mesurés, soit 4,7 p. 100 de l'ensemble des arbres échantillonnés. Ce pourcentage peut être considéré comme représentatif de la proportion réelle d'hybrides dans les populations étudiées, car le biais introduit lors de la stratification de l'échantillonnage reste faible. Nous sommes ainsi en accord avec GATHY (1969) qui considère lhybridation comme un phénomène rare, accidentel et à pourcentage d'apparition très faible par rapport au temps de cohabitation. Jones (1959) pense qu'il est inhabituel de rencontrer plus de 5 p. 100 d'hybrides dans une population. mélangée (bien qu'il en recense lui-même trois sur vingt-cinq individus, à Bagley Wood). MAYER (1977, in BEER, 1981), comme JONEs (loc. cit.), supposent que la compréhension imparfaite des caractères spécifiques plus variables qu'on ne le pense habituellement, expliquerait que certaines études aient déterminé des proportions très importantes d'hybrides en populations naturelles. Cette variabilité pourrait être bien sûr d'origine génétique ou phénotypique. Notons que KraHI-URBaN et al. (1955) ont mis en évidence une forte variabilité intraspécifique des agglutinines des feuilles et fruits selon la provenance.

Pour leur part, Carlisle \& Brown (1965), Cousens (1965), Wigston (1974), Rushton (1978) en Angleterre, Kissling (1980 b) en Suisse, Olsson (1975 a) en Suède, mettent en évidence de larges phénomènes d'hybridation introgressive dans les populations de chênes qu'ils étudient. Notons que tous ont employé des méthodes d'études peu objectives (index d'hybridité, métroglyphes, ...), excepté Rushton qui utilise une analyse multivariable. Il n'étudie cependant que des caractères foliaires, en supposant ceux-ci bien corrélés aux caractères des infrutescences. Or c'est bien souvent en raison des contradictions entre caractères foliaires et caractères des infrutescences que les botanistes ne peuvent déterminer un chêne avec sûreté !

Les essais d'hybridation artificielle devraient permettre de cerner limportance naturelle du phénomène. Or, si les tentatives de Dengler (1941, in Rushton, 1977) ont été variablement réussies ( 0 à 2 p. 100 de réussite, mais 15 p. 100 en 1936 pour le croisement Quercus robur o $\times$ Quercus petraea ô), celles de Rushton (1977) ont montré un très faible taux de réussite interspécifique $(0,6$ p. 100) devant le taux intraspécifique $(39,9$ p. 100). De plus les glands issus de ces croisements sont plus petits que ceux des espèces parentales (résultat confirmé par GATHY, 1969) et non viables, infirmant toute hypothèse d'hétérosis.

Nous a vons retrouvé dans notre étude les caractères discriminants mis en avant par la plupart des auteurs, en les hiérarchisant : longueur du pédoncule floral, longueur du pétiole, degré de développement des lobes foliaires basaux. Les caractères mesurés sur les giands et cupules ne montrent pas de liaison significative avec le statut taxonomique des individus, confirmant des résultats similaires (Jones, 1959; BECKER, 1972; Rushton, 1977). La forme des glands serait donc surtout le résultat de l'action des facteurs externes. Il en est de même pour la forme du rhytidome qui varie indépendamment de l'espèce. Ce dernier résultat n'infirme pas l'observation courante selon laquelle le chêne pédonculé a un rhytidome plus épais et plus profondément fissuré que le chêne sessile. Par contre, il confirme que cette différence peut être totalement masquée par divers facteurs dont la vitesse de croissance de l'arbre, liée elle-même à la sylviculture.

De façon pragmatique, l'étude du diagramme de la figure 5 permet de définir les limites de variation des caractères pour chaque espèce, dans la zone étudiée : le chêne sessile est caractérisé par un pédoncule de longueur totale inférieure à $20 \mathrm{~mm}$ 
(ou, jusquau premier gland, $15 \mathrm{~mm}$ ), de longueur de pétiole supérieure à $9 \mathrm{~mm}$ et avec un angle du lobe de base le plus développé inférieur à 70". Cette dernière valeur est nettement supérieure à celle couramment utilisée et explique en partie la forte proportion d' "hybrides» répertoriés sur le terrain au début de notre étude. Pour ce caractère, le chêne sessile serait donc sujet à une plus large variabilité que nous le supposions a priori. Le chêne pédonculé, de longueur de pédoncule totale supérieure à $20 \mathrm{~mm}$, a une longueur de pétiole inférieure à $5 \mathrm{~mm}$ et un angle d'oreillette supérieur à $140^{\circ}$. Les hybrides éventuels sont à rechercher parmi les arbres ayant une longueur moyenne de pédoncule de $20 \mathrm{~mm}$ environ, et des longueurs de pétiole et angles des oreillettes intermédiaires avec les valeurs précédentes (respectivement 5 à $9 \mathrm{~mm}$ et 70 à $\left.140^{\circ}\right)$.

Ainsi de nombreux auteurs classent a priori les chênes à longueur de pédoncule comprise entre 20 et $30 \mathrm{~mm}$ dans la catégorie «hybride». Selon notre critère, ils deviendraient partie intégrante de l'espèce chêne pédonculé. Il est intéressant de citer à ce sujet les résultats de DERET VARCIN (1983). Sur la base d'une étude morphologique ayant délimité trois types de chênes, sessile (longueur de pédoncule jusquaà la première cupule nullc), pédonculé (Iongueur supérieure à $40 \mathrm{~mm}$ ) et intermédiaire (longueur comprise entre 20 et $30 \mathrm{~mm}$ ) (PELlecuer, 1976), elle étudie les caractéristiques technologiques du bois de chacun des types : pour aucun des caractères étudiés le chêne de type hybride ne se sépare du pédonculé.

Rappelons enfin que nos résultats sont valables sous réserve que les chênes étudiés n'aient pas été directement plantés. Ceci ne semble pas être le cas : en effet, les deux espèces se répartissent le long du transect selon deux gradients opposés. Le chêne pédonculé est le plus fréquent dans les groupements végétaux du Carpinion bétulii, au bas du transect, le chêne sessile le relayant vers le sommet, dans les groupements du Quercion rohori-petraese.

\section{Conclusion}

Notre étude nous a permis de délimiter de façon précise deux unités taxonomiques, avec moins de 5 p. 100 d'individus intermédiaires que lon peut attribuer aux phénomènes d'hybridité.

Nous pensons, sans nier l'évidence de ces phénomènes d'hybridation-introgression chez le chêne, quil faut limiter leur généralité et leur portée quant à lexpression de caractères morphologiques intermédiaires dans les populations naturelles. Ceux-ci pourraient, pour une grande part, être le fait de variations intraspécifiques. La confirmation de ces résultats demande l'étude d'un grand nombre de populations. Il serait donc intéressant d'étendre ce type d'étude biométrique, relativement simple, à des provenances géographiques multiples tant en milieu naturel qu'en plantations comparatives.

Parallèlement, la génétique des isozymes devrait permettre de trancher plus nettement le problème. Otsson (1975 b), étudiant des isopéroxydases, n⿳a pu en trouver le déterminisme génétique, mais a du moins montré une grande hétérogénéité des populations, pour chaque espèce. 


\section{Summary}

Multivariate analysis of some morphological characters of oak (Quercus robur L. and Quercus petraea (Matt.) Liebl.) populations in the Hurepoix (France)

The variability of morphological characters of leaves, acorns and barks is analysed within two mixed populations of pedunculate and sessile oaks. Fifteen characters, divided into fifty-three total classes, were measured or assessed on a sample of fourty-three trees. Reciprocal averaging wich appear to be the most objective method permit a net distinction between trees belonging to the Quercus petraea taxon and these of the Quercus robur taxon. Differential characters are peduncle length, petiole length and auricles angle. None significant difference is found between the two species for the characters of acorns, acorn-cups and barks under study. Less than $5 \%$ of morphological intermediate trees indicates the weakness of the phenomena of introgressive hybridation in this sample. Generality of this result is discussed.

\section{Références bibliographiques}

Baule A., 1980. Etude préalable à l'aménagement des bois du Campus d'Orsay (Essonne). D.E.A. d'Ecologie végétale, Université de Paris-Sud, 22 p. + ann.

Becker M., 1972. Quelques observations morphologiques chez le chêne sessile ct chez le chêne pédonculé. Bull. Soc. bot. Fr., 119, 231-236.

Becker M.. Levy G., 1982. Le dépérissement du chêne en forêt de Tronçais. Les causes écologiques. Ann. Sci. for., 39 (4), 439-444.

BEER R, 1981. Comportement écologique et sylvicole des chênes pédonculés (Quercus robur) et rouvre (Quercus petraea) dans le canton de Genève : conséquences sur le choix des essences pour l'amélioration de la chênaie genevoise. Saussurea, 12, 91-116.

Carlisle A., Brown A.H.F., 1965. The assessment of the taxonomic status of mixed oak (Quercus spp.) populations. Watsonia, 6 (2), 120-127.

Cousens J.E., 1965. The status of the Pedunculate and Sessile oaks in Britain. Watsonia, 6 (3), 161-176.

Deret Varcin E., 1983. Etude comparative de la qualité du bois de trois types de chênes (rouvres, pédonculés et intermédiaires), en forêt de Morimond. Ann. Sci. for., 40 (4) (à paraître).

Gardiner A.S., 1970. Pedunculate and Sessile oak (Quercus robur L. and Quercus petraea (Mattuschka) Liebl.). A review of the hybrid controversy. Forestry, 43 (2), 151-160.

Gathy P., 1969. Contribution à l'étude de la génétique des chênes (Qucrcus sessiliflora Salisb. et Quercus pedunculata Ehrh.). $2^{\text {e }}$ consultation mondiale sur l'amélioration des arbres forestiers, F.A.O., 2, 979-987.

JONES E.W., 1959. Biological Flora of the British Isles. Quercus L. J. Ecol., 47, 169-222.

Kissling P., 1980 a. Clef de détermination des chênes médiocuropéens (Quercus L.). Ber. schweiz. bot. Ges., $90(1 / 2), 29-44$.

KissLING P., 1980 b. Un réseau de corrélation entre les chênes (Quercus) du Jura. Ber. schweiz. bot. Ges., $90(1 / 2), 1-28$.

Krahl-Urban J., Kanitz H.R., Punin W., 1955. Uber den Nachweis von Agglutininen an Früchten und Blättern der Stiel = und Traubeneiche. (Quercus pedunculata Ehrh. und Quercus sessiliflora Salisb.). Z. Forstgenetik, 4 (1), 18-20.

Mathiev A., 1897. Flore forestière. $4^{\circ}$ édit. Paris-Nancy, 705 p.

Olsson U., 1975 a. A morphological analysis of phenotypes in populations of Quercus (Fagaceae) in Sweden. Bot. Not., 128, 53-68. 
Olsson U., 1975 b. Peroxydase isozymes in Quercus petraea and Quercus robur., Bot. Not., 128, 408-411.

Pellecuer B., 1976. L'écologie des chênes en forêt de Morimond et ses applications sylvicoles. Thèse Doct. Ing., Université de Nancy, 184 p.

Poskin A., 1934. Le chêne pédonculé et le chêne rouvre. Leur culture en Belgique. Ed. Duculot, Gembloux, 283 p.

Rushron B.S., 1974. The origin and possible significance of variation of leaf structure in two native British oak species. Quercus robur L. and Quercus petraea. (Matt.) Liebl. D. Phil. Thesis., University of York.

Rushton B.S., 1977. Artificial hybridization between Quercus robur L. and Quercus petraea (Matt.) Liebl. Watsonia, 11, 229-236.

Rushton B.S., 1978. Quercus robur L. and Quercus petraea (Matt.) Liebl. : a multivariate approach to the hybrid problem, 1 : Data acquisition, analysis and interpretation. Watsonia, 12, 81-101.

Turbang J., 1954. Contribution à l'étude de la régénération naturelle du chêne en Lorraine Belge. Bull. Inst. Agron. Gembloux, 90-134.

Wigston D.., 1974. Cytologgy and genetics of oaks in the British oak. Ed. The Botanical Society of the British Isles, 27-50. 